\title{
Experimental and Theoretical Analysis of
}

\section{Self-Pulsation in Distributed Bragg Reflector}

\section{Semiconductor Lasers}

\author{
J. Renaudier*, G.-H. Duan, Senior Member, IEEE, P. Landais ${ }^{\dagger}$, Member, IEEE, \\ B. Thedrez, and P. Gallion ${ }^{\ddagger}$, Senior Member, IEEE
}

\begin{abstract}
In this article, self-pulsation (SP) in a distributed Bragg reflector semiconductor (DBR) laser without saturable absorber is experimentally and theoretically investigated. Detailed presentation of the device structure is given. A complete experimental investigation of the (SP) has demonstrated phase correlation between the longitudinal modes selected by the DBR mirror. The origin of the self-pulsation has been investigated theoretically. A model based on the rate equations of three coupled modes and carrier density has been developed to study the time evolution of phases and amplitudes of the modes. The carrier density modulation, resulting from the beating between adjacent longitudinal modes, generates four wave-mixing. This four-mixing process is responsible for mutual injection locking, leading to passive mode-locking. The calculated power spectral density of frequency noise derived from the model is in agreement with experimental results and proves that the phases of the longitudinal modes are identically correlated through the four-wave-mixing in this type of self-pulsating laser.
\end{abstract}

${ }^{*}$ J. Renaudier, G.-H. Duan, and B. Thedrez are with Alcatel Thales III-V Lab, Alcatel Research \& Innovation, Route de Nozay, 91460 Marcoussis, France.

${ }^{\dagger} \mathrm{P}$. Landais is with the School of Electronic Engineering, Dublin City University, Glasnevin, Dublin 9, Ireland.

${ }^{\ddagger} \mathrm{P}$. Gallion and also J. Renaudier are with the Département Communications et Electronique, CNRS, LCI UMR 514, Ecole Nationale Supérieure des Télécommunications, 46 rue Barrault,75634 Paris Cedex 13, France. 
Index Terms : Distributed Bragg reflector lasers, passive mode-locked lasers, self-pulsating lasers, four wave mixing, and phase synchronization.

\section{Introduction}

In order to improve transmission distance, transparency, capacity and speed of optical networks, much attention has been paid to investigate practical means for all-optical signal processing, for example all-optical digital logic functions and 3R (retiming, reshaping and reamplifying) regenerators [?], [?]. All-optical clock recovery regeneration at $40 \mathrm{Gbit} / \mathrm{s}$ and beyond appears to be a crucial element for future transparent networks. One solution to achieve the regeneration is an all-optical clock recovery element combined with a Mach-Zehnder interferometer [?]. In this respect truly all-optical clock recovery is of very high interest as it would supersede the complicated optoelectronic schemes including: a high speed photo-receiver, a high-Q filter, a power amplifier and a high speed laser or an integrated laser modulator. Among the different approaches investigated so far, we can mention in particular the one using self-pulsating (SP) lasers. Self-pulsation (SP) is a periodic variation of the output power of the laser even though it is DC biased. For instance, SP has been observed in Fabry-Perot [?], [?], distributed feedback (DFB) [?]- [?] and distributed Bragg reflector (DBR) [?]- [?] semiconductor lasers. The FabryPerot type SP laser is not convenient for all-optical clock recovery applications, as the wavelength of the recovered clock is the same as that of the input data. On the contrary, DFB type SP laser with several configurations has been demonstrated for all-optical clock recovery: phasecomb [?], [?], active mirror [?], and gain-coupled [?], [?]. Both phase-comb and gain-coupled DFB SP lasers contain two sub DFB sections and a phase section. Each sub-section has a specific corrugation step. The self-pulsation originates from the beating between these two longitudinal modes co-existing in the cavity. However, such type of SP lasers is quite complexe in terms of fabrication process and behavior.

The other option is the DBR-type laser structure. There are two types of SP DBR lasers: with [?], [?] and without saturable absorber [?], [?]. In DBR lasers with saturable absorber, the SP originates from passive Q-switching own to the modulation of absorption. The non- 
linearities and characteristic times of semiconductor devices lead to oscillation at frequencies up to $500 \mathrm{GHz}$ in this type of DBR lasers [?]. In DBR lasers without saturable absorber, the beating between the longitudinal modes generates a power oscillation under appropriate bias conditions. All-optical clock recovery at $40 \mathrm{Gbit} / \mathrm{s}$ [?] and $160 \mathrm{Gbit} / \mathrm{s}$ [?] has been demonstrated with both types of SP DBR structure. However, nor detailed characteristics of SP DBR without saturable absorber has been reported so far, neither the origin of SP in such DBR lasers has yet been clearly identified. The aims of this paper are two-folded: Firstly it is to report on the design of the SP DBR laser without saturable absorber and its performances as a multimode laser and a $40 \mathrm{GHz}$ wave generator; and secondly to understand the origin of the self-pulsation in such a device. This endeavor is carried out experimentally and theoretically.

The paper is organized as follows: Part ?? describes the details on the laser structure and its SP behavior; Part ?? is devoted to experimental characterisation of the phase correlation between the longitudinal modes; Part ?? develops the model, taking into account the evolution of the amplitude and the phase of the complex electric field of each longitudinal mode. From this model, the phase correlation of these modes is calculated. A conclusion is finally given at the end of the paper.

\section{Laser description and characterisation}

Typical DBR lasers designed to self-pulsate consist of three sections: active, phase and Bragg sections. The particularity of SP DBR lasers, compared to those designed for other applications such as tunable lasers, is the need to operate in multimode regime. Thus, the DBR laser under study, shown in Fig.??, has a short Bragg section of $150 \mu \mathrm{m}$ allowing to have mainly 3 longitudinal modes. The corrugation step of the Bragg reflector is XXnm resulting to a Bragg wavelength of YYYnnm. The active section is $900-\mu \mathrm{m}-$ long and consists of six quantum wells and five barriers of thickness of $8 \mathrm{~nm}$ and $10 \mathrm{~nm}$ respectively, surrounded by two separated confinement heterostructure guiding layers of total thickness of $200 \mathrm{~nm}$. The 1.5 $\mu \mathrm{m}$ width of the waveguide was chosen to ensure a single transverse mode of the electric field. The phase section is $100-\mu \mathrm{m}$ long, with a waveguide width expanding linearly up to the $1.8-\mu \mathrm{m}$ 
width in the Bragg section. This section was designed to adapt the optical modes between the active and the passive optical waveguides. It is kept unbiased for all experiments reported in this article since it is only used for a fine tuning of the emitted wavelength. The different sections are electrically isolated $(\sim 1 \mathrm{M} \Omega)$ by ion implantation. The injected currents in active and Bragg sections are labelled $I_{a}$ and $I_{B}$ respectively. Under large injection currents, such a DBR laser features a multimode longitudinal spectrum. The beating between these modes generates SP at frequency given by the mode-spacing frequency :

$$
\nu_{s p}=\frac{c}{2\left(n_{g} L_{a}+n_{\varphi} L_{\varphi}+n_{B} L_{e f f}\right)}
$$

where $c$ is the velocity of light, $L_{a}, L_{\varphi}$ and $L_{e f f}$ are the active, phase and effective lengths [?] respectively, $n_{g}, n_{\varphi}$ and $n_{B}$ being the gain, phase and Bragg group indices respectively.

To characterize SP from DBR lasers, the output light is lunched into a single mode fiber, passes through an isolator $(>70 \mathrm{~dB})$ to avoid feedback disturbance, and is then analyzed with a slow response power meter, a $0.07 \mathrm{~nm}$ resolution bandwidth optical spectrum analyzer and a $50 \mathrm{GHz}$ bandpass photodiode plugged onto a $300 \mathrm{kHz}$ bandwidth electrical spectrum analyzer (ESA). Fig. ?? shows an example of multimode optical spectrum for driving DC bias currents of $I_{a}=230 \mathrm{~mA}$ and $I_{B}=120 \mathrm{~mA}$. The phase section is set to $0 \mathrm{~mA}$. This optical spectrum clearly reveals that the laser operates with three dominant longitudinal modes separated by about $0.33 \mathrm{~nm}$, corresponding to $\mathbf{S P}$ frequency of $39 \mathrm{GHz}$ approximately. Fig. ?? shows the electrical spectrum of photocurrent observed under the same bias condition than that of Fig. 2. The electrical spectrum is characterized by Lorentzian lineshape centered on a frequency close to $39 \mathrm{GHz}$, with a full width at half-maximum of $1.5 \mathrm{MHz}$ approximately.

Fig. ?? shows the wavelength and power of the main optical mode coupled to a single mode fiber as a function of the active current, with unbiased Bragg section. The threshold current is estimated at $15 \mathrm{~mA}$, and power saturation due to thermal effects occurs around $\mathbf{2 2 5} \mathbf{~ m A}$. The main mode wavelength is red-shifted as the current increases. This is due to the refractive index temperature dependence. The mode hoppings to a longer wavelength observed at 50 and 170 $m A$ suggest a faster increase of the Bragg wavelength compared to that of the cavity modes, due 
to again thermal effects produced by the active current on the laser cavity.

The evolution of the SP frequency versus the active current above $100 \mathrm{~mA}$ is shown in Fig. ??. Values of the SP frequency increase as $I_{a}$ increases. This is still due to the increase of the detuning between the Bragg wavelength and the central wavelength of the two dominant modes. The frequency difference of the two main modes is thus the SP frequency observed. With the increase of the injection current to the active section, the detuning between the Bragg wavelength and that of the middle of the two main modes decreases, leading to a shorter effective length, and thus to a larger frequency spacing between the two dominant modes. Figs. ?? and ?? display the wavelength evolution of the main optical mode as a function of the Bragg current, with an active current of $200 \mathrm{~mA}$. We can see on these figures the typical phenomenon of mode hopping in DBR lasers, where the magnitude of each mode jump corresponds to the mode-spacing. In Fig. ??, as the Bragg current is relatively low, the thermal effects can be neglected. The increase of carrier density in the Bragg section reduces the effective refractive index of this section, resulting in a blue-shift of the lasing mode and mode-hopping to shorter wavelengths. In Fig. ??, as the Bragg current exceeds $50 \mathrm{~mA}$, the main mode now hopes to a longer wavelength. This is attributed to the red shift of the Bragg wavelength due to the increase of Bragg effective index by thermal heating.

These distinct behaviors are still observed on Figs. ?? and ??, showing the SP frequency as a function of the Bragg current. One can observe that the SP frequency jumps accordingly to mode jumps, and between two jumps, the SP frequency decreases on Fig. ?? and increases on Fig. ??. Again the SP frequency variation is attributed to the detuning variation between the center of the two main modes and the Bragg wavelength. 


\section{Experimental demonstration of phase correlation between lon- gitudinal modes}

As demonstrated in the previous section, there is an interplay between the optical spectrum and the value of optical self-pulsation frequency.

To understand the observed SP phenomenon, each longitudinal mode is individually selected by a $0.1 \mathrm{~nm}$ bandpass filter and its spectral linewidth is measured using a self-heterodyne technique based on a Mach-Zehnder interferometer. These linewidths are then compared to that of the SP signal measured on the ESA. Fig. ?? shows the spectral linewidths of longitudinal modes and SP signal as a function of the Bragg current with an active current fixed to 200 mA. This measurement was performed between two mode jumps, see Figure 6(a). It is observed that the spectral linewidths of the longitudinal modes decrease with increasing current. Such a decrease was attributed to the decrease of the effective phase-amplitude coupling factor [?]. Since SP is generated by the beating between these modes, the linewidth of the RF signal is also affected by the increase of Bragg current and follows the decrease of the linewidths of the longitudinal modes. Furthermore, we can easily see that the linewidth of the RF signal from SP is always largely narrower than that of the sum of the longitudinal modes as in FP semiconductor lasers [?]. The results demonstrates the phase of each longitudinal mode fluctuates upon spontaneous emission, with a linewidth of the order of several tens of MHz. However, the reduced linewidth of the SP signal, of the order of $\mathrm{MHz}$, shows that these fluctuations are largely synchronized. This proves that the self-pulsations observed does not results from a beating at the level of the photodiode, but it is an intra-cavity phenomenon.

In the following experiment, the active region is biased at $200 \mathrm{~mA}$, the Bragg and phase sections are unbiased. Using the same experimental setup than that of Part ??, the radiofrequency $(\mathrm{RF})$ spectrum of the photocurrent is measured and it is displayed in Fig. ??. The photocurrent features a Lorentzian shape centered at $39 \mathrm{GHz}$ with a full width at half-maximun of $700 \mathrm{kHz}$, approximately. The corresponding optical spectrum is shown in the insert. The 
laser operates in a three mode regime: the strongest is labelled number 2 , and the next modes respectively on the right and on the left are labelled number 1 and number 3 . The other modes, at least $10 \mathrm{~dB}$ lower in power than the third one.

The optical signal is launched into a monochromator acting like a tunable optical bandpass filter. Setting the bandwidth to $0.5 \mathrm{~nm}$, the monochromator enables to select two pairs of modes, i.e. $1 \& 2$ or $2 \& 3$. optical modes. The filtered optical signal is directly led to the photodiode followed by the ESA to characterize the two-modes beating. Fig. ?? displays the RF spectrum of the photocurrent representing the beating between modes $1 \& 2$ that are represented in the insert. We observe that this spectrum also appears as a Lorenztian shaped signal, with a maximum peak power largely reduced compared with the one of Fig. ??. This $25 \mathrm{~dB}$ reduction corresponds to the insertion losses due to the monochromator. The measured full width at half maximum is about $710 \mathrm{kHz}$, which is close to the spectral linewidth of the entire SP signal. Similarly, the RF spectrum from the beating component of modes 2 \& 3 is shown in Fig. ??. The insert shows the corresponding filtered optical spectrum. The full width at half-maximun is about $730 \mathrm{kHz}$. The Lorentzian shapes generated by the beatings between modes $1 \& 2$ and between modes $2 \& 3$ have the same spectral linewidths. This experiment demonstrates that whole SP of Fig. 9 is composed of at least two beatings with the same value for RF linewidths. More fundamentally, it demonstrates that phases of modes 1 and 3 are identically correlated to the phase of the mode 2 . Since phase noise is at the origin of spectral linewidth, such measurements proves that the relative phases between adjacent modes present the same phase noise characteristics, suggesting that the modes are partly correlated under passive mode-locking (PML) operation.

The next part addresses a theoretical model developed to demonstrate that four-wave-mixing is largely responsible for phase synchronization under PML in DBR semiconductor lasers. 


\section{Theoretical model}

In order to understand the observed SP, and in particular, to identify the origin of SP in such a multimode laser, a model has been developed based on the time evolution of multiple optical fields generated inside the DBR laser. In this model, we consider that the cavity waveguide supports only the fundamental TE mode with the transverse distribution $U(x, y)$. The monochromatic wave representing the optical field of the $k^{t h}$ mode is expressed as follows:

$$
\epsilon_{k}(x, y, z, t)=E_{k}(t) \exp \left(-\imath \omega_{k} t\right) \cdot U(x, y) \cdot Z_{k}(z)
$$

with the complex amplitude $E_{k}$ defined as:

$$
E_{k}(t)=A_{k}(t) \exp \left(-\imath \phi_{k}(t)\right)
$$

where $A_{k}$ is the slowly time varying amplitude, $Z_{k}$ the longitudinal dependence, $\omega_{k}$ the locked angular frequency and $\phi_{k}$ the instantaneous phase fluctuation. In the case of a laser with $M$ longitudinal modes, the total electric intensity is given by:

$$
\begin{aligned}
\left|\epsilon_{T}(x, y, z, t)\right|^{2}= & \sum_{k=1}^{M}\left|E_{k}(t)\right|^{2}|U(x, y)|^{2}\left|Z_{k}(z)\right|^{2} \\
& +\sum_{k=1}^{M} \sum_{l>k}^{M}\left(E_{k} E_{l}^{*}|U(x, y)|^{2} Z_{k}(z) Z_{l}^{*}(z) \exp \left(\imath \Omega_{l k} t\right)+\text { c.c. }\right),
\end{aligned}
$$

where c.c denotes the complex conjugate of the proceeding term, and $\Omega_{l k}=\omega_{l}-\omega_{k}$. For a laser with two uncorrelated modes, a RF signal at the frequency $\Omega_{l k} / 2 \pi$ can be observed by a photodiode, with a spectral linewidth corresponding to the sum of the modes' spectral linewidths. In the SP regime, all modes generated inside the laser cavity are passively locked such that all mode-spacing angular frequencies are fixed to the so-called SP angular frequency $\Omega_{s p}$. By setting $\Omega_{l k}=(l-k) \Omega_{s p}=m \Omega_{s p}$ in Eq. (??), the total light intensity can be written as:

$$
\begin{aligned}
\left|\epsilon_{T}(x, y, z, t)\right|^{2} & =\left|\epsilon_{T 0}(x, y, z, t)\right|^{2} \\
& +\sum_{m=1}^{M-1}\left(\sum_{k=m+1}^{M} E_{k} E_{k-m}^{*}|U(x, y)|^{2} Z_{k}(z) Z_{k-m}^{*}(z)\right) \exp \left(-\imath m \Omega_{s p} t\right)+\text { c.c. }
\end{aligned}
$$

with $\left|\epsilon_{T 0}(x, y, z, t)\right|^{2}$ being the time average photon density, corresponding to the first term of Eq. (??). This equation shows that the total light intensity contains the beating terms with 
several harmonics.

\subsection{Carrier density modulation}

The beating between the different modes creates a gain and index modulation through five nonlinear effects in an active semiconductor medium: the carrier density modulation (CDM), the carrier heating, the spectral hole burning, the two-photon absorption and the Kerr effect [?]. In the present paper, only CDM will be taken into account, as it is the dominant effect compared to other four effects for mode spacing of the order of $40 \mathrm{GHz}$ [?]. However, the model presented here can be easily generalized to take into account other four effects. The origin of CDM in multimode semiconductor lasers is the interaction between linear gain and the total electric field, as described by the carrier density rate equation below:

$$
\frac{d N(x, y, t)}{d t}=\frac{}{e V}-\frac{N(x, y, t)}{\tau_{e}}-v_{g} g_{d}\left(N(x, y, t)-N_{t r}\right) \cdot\left|\epsilon_{T}(x, y, t)\right|^{2}
$$

where the electrical field $\epsilon_{T}(x, y, t)$ is scaled such that $\left|\epsilon_{T}(x, y, t)\right|^{2}$ represents the photon density of the $k^{t h}$ mode inside the laser cavity, $\mathrm{V}$ is the volume of the active layer, e is the electron charge, $\tau_{e}$ is the carrier lifetime, $v_{g}$ is the group velocity, $g_{d}$ is the differential gain and $N_{t r}$ is the carrier density at transparency.

The mode beating leads to the carrier density modulation of the form:

$$
N(x, y, t)=N_{0}(x, y, t)+\sum_{m=1}^{M-1}\left(N_{m} \exp \left(-\imath m \Omega_{s p} t\right)+\text { c.c. }\right)
$$

By introducing Eqs. (??) and (??) in Eq. (??), the following expression of the first order for $N_{m}$ is found [?]

$$
N_{m}=|U(x, y)|^{2} \Delta N_{m}, \quad \Delta N_{m}=-\left(N_{0}-N_{t r}\right) \cdot \frac{\sum_{k=m+1}^{M} E_{k} E_{k-m}^{*} / P_{s}}{1+P_{t} / P_{s}-\imath m \Omega_{s p} \tau_{e}},
$$

whith the photon saturation density $P_{s}=1 /\left(\Gamma v_{g} g_{d} \tau_{e}\right)$, and the local photon density $P_{t}=$ $\sum_{k=1}^{M}|U(x, y)|^{2}\left|E_{k}\right|^{2}$. Since $\Omega_{s p} \tau_{e} \gg 1$, the term $\left(1+P_{t} / P_{s}\right)$ can be neglected compared to $\imath m \Omega_{s p} \tau_{e}$. The spatial average carrier density $\bar{N}_{0}$ sastisfies the following rate equation:

$$
\frac{d \bar{N}_{0}}{d t}=\frac{\bar{N}_{0}}{e V}-G \bar{P}_{t}
$$


where the overbar denotes averaging in spatial dimensions. $\bar{P}_{t}$ is the average photon density, the linear gain is $G=\Gamma v_{g} g_{d}\left(\bar{N}_{0}-N_{t r}\right)$, and $\Gamma$ is the confinement factor defined as:

$$
\Gamma=\frac{\int_{-d / 2}^{d / 2} \int_{-w / 2}^{w / 2}|U(x, y)|^{2} d x d y}{\int_{-\infty}^{\infty} \int_{-\infty}^{\infty}|U(x, y)|^{2} d x d y}
$$

with $w$ and $d$ being respectively the width and the thickness of the active waveguide.

\subsection{Coupled rate equations}

CDM leads to a nonlinear gain and refractive index modulation, affecting both the amplitude and the phase of the lasing modes. Corresponding to our experiments and for the sake of simplicity and clarity, only three longitudinal modes $(M=3)$ propagating in the SP DBR are considered in our approach. We start from rate equations of the $k^{\text {th }}$ passively locked mode for semiconductor lasers including the small-signal expression of carrier density in Eq. (??) [?]:

$$
\begin{aligned}
\frac{d E_{k}}{d t}= & \frac{1}{2}\left(1-\imath \alpha_{H}\right)\left(G-\gamma_{k}\right)-\imath\left(\omega_{k}^{i}-\omega_{k}\right) E_{k} \\
& +\frac{1}{2}\left(1-\imath \alpha_{H}\right) v_{g} g_{d} \Gamma C \times\left(\sum_{m=1}^{M-1}\left(\Delta N_{m} E_{k-m}+\Delta N_{m}^{*} E_{k+m}\right)\right),
\end{aligned}
$$

where $\alpha_{H}$ represents the phase-amplitude coupling factor, $\gamma_{k}$ the cavity losses for the $k^{\text {th }}$ mode. The linear gain, $G$, is assumed constant over the spectral range of the three modes. The occurrence of the term $\left(\omega_{k}-\omega_{k}^{i}\right)$ accounts for the detuning of the locked angular frequency $\omega_{k}$ of the $k^{t h}$ lasing mode from its cavity resonance one $\omega_{k}^{i}$. C is the overlap factor, given by:

$$
C=\frac{\int_{-d / 2}^{d / 2} \int_{-w / 2}^{w / 2}|U(x, y)|^{4} d x d y}{\int_{-d / 2}^{d / 2} \int_{-w / 2}^{w / 2}|U(x, y)|^{2} d x d y}
$$

It was first introduced by Bogatov [?] and Agrawal [?], and takes into account the influence of the transverse contribution to non-linear gain.

Eq. (??) is similar to those already developed by Sargent [?] and Shore [?] to describe modelocking and coupling in multi-mode lasers. However, in our model, we concentrate on the effect of carrier density modulation resulting from the beating of longitudinal modes selected by the DBR mirror to determine the origin of SP. By separating real and imaginary parts of Eq. (??), six rate equations are obtained, describing the time evolution of both the amplitude and the 
phase of the three modes:

$$
\begin{aligned}
\frac{d A_{1}}{d t} & =\frac{G}{2}\left(1+\vartheta_{c d m}\left(\frac{\alpha_{H}}{2} \frac{A_{3}^{2}}{P_{s}}+\alpha_{H} \frac{A_{2}^{2}}{P_{s}}+\sqrt{1+\alpha_{H}^{2}} \frac{A_{2}^{2}}{P_{s}} \frac{A_{3}}{A_{1}} \sin \left(\psi+\arctan \alpha_{H}\right)\right)\right) A_{1}-\frac{\gamma_{1}}{2} A_{1} \\
\frac{d A_{2}}{d t} & =\frac{G}{2}\left(1-\vartheta_{c d m} \alpha_{H}\left(\frac{A_{1}^{2}}{P_{s}}-\frac{A_{3}^{2}}{P_{s}}\right)\right) A_{2}-\frac{\gamma_{2}}{2} A_{2} \\
\frac{d A_{3}}{d t} & =\frac{G}{2}\left(1-\vartheta_{c d m}\left(\frac{\alpha_{H}}{2} \frac{A_{1}^{2}}{P_{s}}+\alpha_{H} \frac{A_{2}^{2}}{P_{s}}+\sqrt{1+\alpha_{H}^{2}} \frac{A_{2}^{2}}{P_{s}} \frac{A_{1}}{A_{3}} \sin \left(\psi+\arctan \alpha_{H}\right)\right)\right) A_{3}-\frac{\gamma_{3}}{2} A_{3} \\
\frac{d \phi_{1}}{d t} & =\left(\omega_{1}-\omega_{1}^{i}\right)+\frac{G}{2}\left(\alpha_{H}-\vartheta_{c d m}\left(\frac{1}{2} \frac{A_{3}^{2}}{P_{s}}+\frac{A_{2}^{2}}{P_{s}}+\sqrt{1+\alpha_{H}^{2}} \frac{A_{2}^{2}}{P_{s}} \frac{A_{3}}{A_{1}} \cos \left(\psi+\arctan \alpha_{H}\right)\right)\right)-\frac{\gamma_{1}}{2} \\
\frac{d \phi_{2}}{d t} & =\left(\omega_{2}-\omega_{2}^{i}\right)+\frac{G}{2}\left(\alpha_{H}+\vartheta_{c d m}\left(\frac{A_{1}^{2}}{P_{s}}-\frac{A_{3}^{2}}{P_{s}}\right)\right)-\frac{\gamma_{2}}{2} \\
\frac{d \phi_{3}}{d t} & =\left(\omega_{3}-\omega_{3}^{i}\right)+\frac{G}{2}\left(\alpha_{H}+\vartheta_{c d m}\left(\frac{1}{2} \frac{A_{1}^{2}}{P_{s}}+\frac{A_{2}^{2}}{P_{s}}+\sqrt{1+\alpha_{H}^{2}} \frac{A_{2}^{2}}{P_{s}} \frac{A_{1}}{A_{3}} \cos \left(\psi+\arctan \alpha_{H}\right)\right)\right)-\frac{\gamma_{3}}{2}
\end{aligned}
$$

where $\vartheta_{c d m}$ quantifies the effect of carrier density modulation, and $\psi$ is the relative phase mismatch, defined respectively as:

$$
\vartheta_{c d m}=\frac{C}{\Omega_{s p} \tau_{e}}, \quad \psi=\left(\phi_{2}-\phi_{1}\right)-\left(\phi_{3}-\phi_{2}\right) .
$$

Amplitude and phase rate equations do not depend on individual phases but on the relative phase mismatch $\psi$ and an additional non-linear terms, expressing either energy or phase transfers between modes. The nonlinear phase-independent terms yield to the gain enhancement of the mode 1 and gain reduction of the mode 3 . The nonlinear phase sensitive terms depends on the relative phase mismatch $\psi$. It is worth noticing that, considering three longitudinal modes in the laser, only beatings at the fundamental pulsation may induce phase transfers. This is the reason why no term of phase transfer appears in the rate equation of the center field $E_{2}$.

\subsection{Mutual injection locking and locking bandwidth}

The beating process among longitudinal modes modulates the carrier density at multiples of the frequency mode spacing. This mode spacing varies from one couple of modes to the other 
in the DBR laser because of the dispersion of the effective length in the Bragg grating. The self-induced carrier density pulsation leads to cross-saturation and FWM through the creation of dynamic index and gain gratings and generates modulation side-bands that act as optical injection signals for modes, as shown in Fig. ??. This effect appears to be a mutual injection locking phenomenon that reaches equilibrium when all mode-spacing frequencies are locked to the same value $\Omega_{s p}$ with appropriate power distribution among modes.

To provide more insight into the mechanism of mutual injection locking, the injection optical fields $E_{i n j, 1}$ and $E_{i n j, 3}$ can be introduced explicitly expressed by:

$$
E_{i n j, 1}=\rho_{i n j, 1} \cdot \exp \left(\phi_{i n j, 1}\right), \quad E_{i n j, 3}=\rho_{i n j, 3} \cdot \exp \left(\phi_{i n j, 3}\right)
$$

with the injection rate $\rho_{i n j, k}$ and the phase $\phi_{i n j, k}$ defined respectively as:

$$
\rho_{i n j, 1}=G \vartheta_{c d m} \sqrt{1+\alpha_{H}^{2}} \frac{A_{2}^{2}}{P_{s}} \frac{A_{1}}{A_{3}}, \quad \rho_{i n j, 3}=G \vartheta_{c d m} \sqrt{1+\alpha_{H}^{2}} \frac{A_{2}^{2}}{P_{s}} \frac{A_{3}}{A_{1}},
$$

and

$$
\phi_{i n j, 1}=2 \phi_{2}-\phi_{1}+\arctan \alpha_{H}, \quad \phi_{i n j, 3}=2 \phi_{2}-\phi_{3}+\arctan \alpha_{H}
$$

These terms describe the injection of optical fields of amplitude $\frac{A_{2}^{2}}{P_{s}} A_{k}(\mathrm{k}=\{1,3\})$ balanced by magnitude of non-linear effects $G \vartheta_{c d m} \sqrt{1+\alpha_{H}^{2}}$. Introducing injection rate terms in the coupled rate equations (??)-(??), we achieve for the amplitudes and the phases the following equations:

$$
\begin{aligned}
& \frac{d A_{1}}{d t}=\frac{G}{2}\left(1+\vartheta_{c d m}\left(\frac{\alpha_{H}}{2} \frac{A_{3}^{2}}{P_{s}}+\alpha_{H} \frac{A_{2}^{2}}{P_{s}}\right)\right) A_{1}+\frac{\rho_{i n j, 3}}{2} \sin \left(\phi_{i n j, 3}-\phi_{1}\right) A_{1}-\frac{\gamma_{1}}{2} A_{1} \\
& \frac{d A_{2}}{d t}=\frac{G}{2}\left(1-\vartheta_{c d m} \alpha_{H}\left(\frac{A_{1}^{2}}{P_{s}}-\frac{A_{3}^{2}}{P_{s}}\right)\right) A_{2}-\frac{\gamma_{2}}{2} A_{2} \\
& \frac{d A_{3}}{d t}=\frac{G}{2}\left(1-\vartheta_{c d m}\left(\frac{\alpha_{H}}{2} \frac{A_{1}^{2}}{P_{s}}+\alpha_{H} \frac{A_{2}^{2}}{P_{s}}\right)\right) A_{3}-\frac{\rho_{i n j, 1}}{2} \sin \left(\phi_{i n j, 1}-\phi_{3}\right) A_{3}-\frac{\gamma_{3}}{2} A_{3}
\end{aligned}
$$




$$
\begin{aligned}
\frac{d \phi_{1}}{d t} & =\left(\omega_{1}-\omega_{1}^{i}\right)+\frac{G}{2}\left(\alpha_{H}-\vartheta_{c d m}\left(\frac{1}{2} \frac{A_{3}^{2}}{P_{s}}+\frac{A_{2}^{2}}{P_{s}}\right)\right)-\frac{\rho_{i n j, 3}}{2} \cos \left(\phi_{i n j, 3}-\phi_{1}\right)-\frac{\gamma_{1}}{2} \\
\frac{d \phi_{2}}{d t} & =\left(\omega_{2}-\omega_{2}^{i}\right)+\frac{G}{2}\left(\alpha_{H}+\vartheta_{c d m}\left(\frac{A_{1}^{2}}{P_{s}}-\frac{A_{3}^{2}}{P_{s}}\right)\right)-\frac{\gamma_{2}}{2} \\
\frac{d \phi_{3}}{d t} & =\left(\omega_{3}-\omega_{3}^{i}\right)+\frac{G}{2}\left(\alpha_{H}+\vartheta_{c d m}\left(\frac{1}{2} \frac{A_{1}^{2}}{P_{s}}+\frac{A_{2}^{2}}{P_{s}}\right)\right)+\frac{\rho_{i n j, 1}}{2} \cos \left(\phi_{i n j, 1}-\phi_{3}\right)-\frac{\gamma_{3}}{2} .
\end{aligned}
$$

The rate equations for side modes 1 and 3, (??), (??) and (??), (??) are similar to those describing an externally injection-locked laser [?]. The only difference with external injection locking phenomenon is that the injection optical fields are self-generated inside the laser cavity. These equations really demonstrate that modulation side-bands created by FWM act as internal injection signal. Fig. ?? shows schematically the mutual injection locking process. For the sake of simplicity, $\omega_{2}$ is considered equal to $\omega_{2}^{i}$ in this schematic description. The effect of modulation side-bands depends on relative power between modes involved ( 1 and 3 here) through injection rate terms. Indeed, the stronger is a modulation side-band, the larger is the frequency shift of the injected mode. This is the reason why the SP frequency is approximately the mode-spacing frequency between the two main modes. Since this phenomenon of mutual injection locking enables the shift of modes' angular frequency from cavity resonance to match the condition of modes passively locked $\left(\omega_{3}-\omega_{2}=\omega_{2}-\omega_{1}=\Omega_{s p}\right)$, our modelling of passive mode-locking including CDM is self-consistent.

In order to determine the validity limit of this system of differential equations (??)-(??), the amplitude and phase time-rate derivations are set to zero. Time variables of amplitude and phase are substituted by their static values written $A_{k}^{0}$ et $\phi_{k}^{0}$. Stationary equations yields to a relation between relative phase mismatch $\psi^{0}$ and initial angular frequency mismatch $\Delta \omega^{i}$ defined as $\left(\omega_{2}^{i}-\omega_{1}^{i}\right)-\left(\omega_{3}^{i}-\omega_{2}^{i}\right)$. Under the SP condition $\left(\omega_{2}-\omega_{1}=\omega_{3}-\omega_{2}\right)$, we obtain the following expression of initial angular frequency mismatch:

$$
\Delta \omega^{i}=\frac{\sqrt{1+\alpha_{H}^{2}}}{2}\left(\rho_{i n j, 1}-\rho_{i n j, 3}\right)\left(\frac{3}{2} \frac{A_{3}^{0} A_{1}^{0}}{A_{2}^{0}}-\cos \left(\psi^{0}\right)\right) .
$$


This expression shows the relationship between the initial angular frequency mismatch $\Delta \omega^{i}$ and the relative phase mismatch $\psi^{0}$. A limit value of the initial angular frequency mismatch for passive mode-locking can then be found:

$$
\left|\Delta \omega^{i}\right|<\frac{5}{4} \sqrt{1+\alpha_{H}^{2}}\left|\rho_{i n j, 1}-\rho_{i n j, 3}\right|
$$

If the initial angular frequency mismatch $\Delta \omega^{i}$ exceeds this limit value, passive mode-locking cannot be achieved. The limit is similar to the locking bandwidth, defined for external optical injection, and is fixed by the injection rate terms [?]. The phase-amplitude coupling factor may induce an enhancement of the maximal detuning between initial frequencies. Besides, when one of the side-modes is stronger than the other, the locking strength increases to induce a larger frequency shift of the last one, as shown in Fig. ??. This figure also shows that, by increasing the photon density inside the cavity (expressed by the ratio $A_{2}^{2} / P_{s}$ ), the locking bandwidth quickly becomes larger than one $\mathrm{GHz}$ for any injection power rate. Considering a free spectral range in the order of $40 \mathrm{GHz}$ for our DBR lasers, such values of locking bandwidth explains why SP appears as soon as the output power reaches $1 \mathrm{~mW}$ in our DBR laser.

\subsection{Calculations of frequency noise spectral densities}

The instantaneous fluctuations of electric fields due to spontaneous emission induce amplitude and phase fluctuations around their static values. The study of phase noise characteristic, which is essential to investigate phase correlation, requires to take such fluctuations into account in our rate equations using Langevin noise sources $F_{A_{k}}, F_{\phi_{k}}$ and $F_{N}$, for amplitude, phase and carrier density respectively. These Langevin sources are characterized by their spectral densities as follows [?], [?]:

$$
\begin{aligned}
& <\widetilde{F}_{A_{k}}(\Omega) \cdot \widetilde{F}_{A_{k}}^{*}\left(\Omega^{\prime}\right)>=\frac{R_{s p}}{2} \cdot \delta\left(\Omega-\Omega^{\prime}\right), \\
& <\widetilde{F}_{\phi_{k}}(\Omega) \cdot \widetilde{F}_{\phi_{k}}^{*}\left(\Omega^{\prime}\right)>=\frac{R_{s p}}{2 A_{k}^{02} V} \cdot \delta\left(\Omega-\Omega^{\prime}\right), \\
& <\widetilde{F}_{N}(\Omega) \cdot \widetilde{F}_{N}^{*}\left(\Omega^{\prime}\right)>=\frac{I}{e V} \cdot \delta\left(\Omega-\Omega^{\prime}\right),
\end{aligned}
$$

where $R_{s p}$ is the spontaneous emission rate, $\delta$ is the Dirac distribution and $\tilde{X}$ denotes the Fourier Transform of the variable $X$. After linearization and Fourier transformation of Eqs. (??), (??)- 
(??), including Langevin noise sources, the following equation is achieved for the angular analysis frequency $\Omega$ :

$$
(j \Omega I-M) \cdot\left(\begin{array}{c}
\delta \widetilde{A}_{1} \\
\delta \widetilde{A}_{2} \\
\delta \widetilde{A}_{3} \\
\delta \widetilde{\phi}_{1} \\
\delta \widetilde{\phi}_{2} \\
\delta \widetilde{\phi_{3}} \\
\delta \widetilde{N}_{0}
\end{array}\right)=\left(\begin{array}{c}
\widetilde{F}_{A_{1}} \\
\widetilde{F}_{A_{2}} \\
\widetilde{F}_{A_{3}} \\
\widetilde{F}_{\phi_{1}} \\
\widetilde{F}_{\phi_{2}} \\
\widetilde{F}_{\phi_{3}} \\
\widetilde{F}_{N}
\end{array}\right)
$$

where $\mathrm{M}$ is $7 \times 7$ matrix and $I$ the unity matrix. For any values of $\Omega$ satisfying the condition that the determinant of $(j \Omega I-M)$ is not equal to zero, the phase fluctuations of the three modes as a function of the Langevin terms can be written as:

$$
Q=(j \Omega I-M)^{-1}=\left(q_{i j}\right)_{\{i, j\} \varepsilon[1,6]},
$$

the phase fluctuations are given directly by:

$$
\delta \widetilde{\phi}_{k}(\Omega)=\sum_{i=1}^{7} q_{k+3, i} \widetilde{F}_{i}(\Omega),
$$

where $k$ varies from 1 to $3, q$ are the elements of matrix $Q$ and $\widetilde{F}_{i}$ is equal to $\widetilde{F}_{A_{i}}$ for $i=1,2,3$, $\widetilde{F}_{\phi_{i}}$ for $i=4,5,6$ and $\widetilde{F}_{N}$ for $i=7$. Using the Eq (??) and the properties of the Langevin sources, the power spectral density (PSD) of the phase noise of the $k^{\text {th }}$ mode can be written as follows:

$$
\begin{aligned}
S_{\phi_{k}}(\Omega) & =<\delta \widetilde{\phi}_{k}(\Omega) \cdot \delta \widetilde{\phi}_{k}^{*}(\Omega)> \\
& =\sum_{i=1}^{7}\left|q_{k, i}\right|^{2}<\widetilde{F}_{i}(\Omega) \cdot \widetilde{F}_{i}^{*}(\Omega)>
\end{aligned}
$$

The PSD of frequency modulation (FM) noise can then be obtained using the following relation:

$$
S_{\dot{\phi_{k}}}(\Omega)=\Omega^{2} S_{\phi_{k}}(\Omega)
$$

In our numerical calculations, the steady operating points are first obtained by Runge-Kutta method. The values of parameters used for these calculations are listed in Table ??. Fig. ?? 
shows the PSD of FM noise of the three individual modes as a function of analysis frequency $f=\Omega / 2 \pi$ for an injected active current of $130 \mathrm{~mA}$, which is roughly equal to ten times the threshold current. The FM noise spectrum the three modes features a peak at the relaxation frequency measured at $9 \mathrm{GHz}$. Assuming a Lorentzian shape for longitudinal modes, the spectral linewidth is given by $S_{\dot{\phi}_{k}}(0) / 2 \pi$, yielding to spectral linewidths of tens of $\mathrm{MHz}$ for each modes. The PSD of relative phases $\phi_{21}$ and $\phi_{32}$ can be calculated by using the relation:

$$
S_{\dot{\phi_{l k}}}(\Omega)=\Omega^{2} S_{\phi_{l k}}(\Omega)
$$

Fig. ?? shows the FM noise PSD of the relative phases, and the sum of FM noise PSD for longitudinal modes, for the same biasing conditions as in Fig. ??. The solid curves describe the FM noise spectra related to modes $1 \& 2$, and the dashed ones describe the FM noise spectra related to modes $2 \& 3$. A dramatic reduction of PSD of the relative phases from that of the sum of FM noise PSD of longitudinal modes for low analysis frequencies can be observed. The calculated values of spectral linewidths are of the same order as those observed experimentally, shown in Fig. 8. Even more, these theoretical values are identical for $\phi_{21}$ and $\phi_{32}$ in a range of several tens of $\mathrm{MHz}$ around analysis frequency, which shows that the correlation level between phases of modes $1 \& 2$ is the same than that between phases of modes $2 \& 3$. This completely agrees with experimental results in Figs. ?? and ?? proving that modes are identically correlated in phase by a FWM process.

\section{Conclusions}

In this paper, self-pulsation in DBR lasers was experimentally and theoretically analyzed. A detailed characterization of the component was presented. The origin of the self-pulsation in such components has been clearly identified: the carrier modulation resulting from the beating of adjacent longitudinal modes selected by the DBR mirror. The theoretical work based on the rate equations of three modes has been developed to study the time evolution of phases and amplitudes of the modes passively locked. It is established that four wave-mixing is responsible for mutual injection locking between lasing modes. It also demonstrates, experimentally and 
theoretically, that the phases of the longitudinal modes are identically correlated through the four-wave-mixing in this type of self-pulsating laser. Our analysis can satisfactorily explain the experimental results obtained from these lasers, and provide guidelines to design high performance SP DBRs for all-clock recovery application.

Acknowledgement: The work presented in this paper is partly supported by the French RNRT "ROTOR" project. 


\section{References}

[1] A. Jourdan "The perspective of optical packet switching in IP dominant backbone and metropolitan networks", IEEE Communication Magazine, vol. 39, pp.136-141, 2001.

[2] B. Lavigne, P. Guerber, P. Brindel, E. Balmefrezol, and B. Dagens, "Cascade of 100 optical 3R regenerators at $40 \mathrm{Gbit} / \mathrm{s}$ based on all-active Mach-Zehnder interferometer", European Conference on Optical Communications, Paper We.F.2.6, pp.290-291, 2001.

[3] L. F. Tiemeijer, P. I. Kuindersma, P. J. A. Thijs, and G. L. J. Rikken, "Passive FM locking in InGaAsP semiconductor lasers", IEEE Journal of Quantum Electronics, vol.25, pp. 1385-1392, 1991.

[4] K. Sato, "Optical pulse generation using Fabry-Perot lasers under continuous wave operation", IEEE Journal of Selected Topics in Quantum Electronics, vol.9, pp. 1288-1293, 2003.

[5] M. Mohrle, B. Sartorius, R. Steingruber, and P.Wolfram, "Electrically switchable selfpulsations in integratable multisection DFB-lasers", IEEE Photonics Technology Letters, vol. 8, pp. $28-30,1996$.

[6] M. Mohrle, B. Sartorius, C. Bornholdt, S. Bauer, O. Brox, A. Sigmund, R. Steingruber, H. Radziunas, and H.- J. Wunsche, "Detuned grating multisection-RW-DFB lasers for highspeed optical signal processing", IEEE Journal of Selected Topics in Quantum Electronics, vol. 7 , pp. 217-223, 2001.

[7] O. Brox, S. Bauer, M. Biletzke, H. Ding, J. Kreissl, H.- J. Wnsche, and B. Sartorius, "Selfpulsating DFB for $40 \mathrm{GHz}$ clock-recovery: impact of intensity fluctuations on jitter", in Proc. Optical Fiber Communication 2004, paper MF55, Los Angeles, 2004.

[8] Y. Li, C. Kim, G. Li, Y. Kaneko, R. L. Jungerman, and O. Buccafusca, "Wavelength and polarization insensitive all-optical clock recovery from $96 \mathrm{~Gb} / \mathrm{s}$ data by using a two-section gain-coupled DFB laser", IEEE Photonics Technology Letters, vol.15, pp. 590-592, 2003. 
[9] W. Mao, Y. Li, M. Al-Mumin, and G. Li, "All-optical clock recovery from RZ-format data by using a two-section gain-coupled DFB laser", Journal of Lightwave Technology, vol. 20, pp. 1705-1714, 2002.

[10] S. Arahira, S. Oshiba, Y. Matsui, T. Kunii, and Y. Ogawa, "500 GHz optical short pulse generation from a monolithic passively mode-locked distributed Bragg reflector laser diode", Applied Physics Letters, vol. 64, No. 15, pp. 1917-1919, 1994.

[11] H. Bao, Y. J. Wen, and H. F. Liu, "Impact of saturable absorption on performance of optical clock recovery using a mode-locked multisection semiconductor laser", IEEE Journal of Quantum Electronics, vol. 40, No. 9, pp. 1177-1185, 2004.

[12] G-H. Duan, C. Gosset, B. Lavigne, R. Brenot, B. Thedrez, J. Jacquet and O. Leclerc, "40 GHz all-optical clock recovery using polarization insensitive distributed Bragg reflector lasers", in Proc. Conference on Lasers and Electro-Optics 2003, paper CThQ5, Baltimore, 2003.

[13] T. Ohno, R. Iga, Y. Kondo, T. Ito, T. Futura, H. Ito and K. Sato, ”Wide-locking-range 160-GHz optical clock recovery from 160-Gbit/s signal using mode-locked laser diode", in Proc. Optical Fiber Communication 2004, paper WD5, Los Angeles, 2004.

[14] Y. Suematsu, S. Arai, and K. Kishino, "Dynamic single-mode semiconductor lasers with a distributed reflector", Journal of Lightwave Technology, vol. LT-1, pp. 161-176, 1983.

[15] G- H. Duan, P. Gallion, and G. Debarge, "Analysis of the phase-amplitude coupling factor and spectral linewidth of distributed feedback and composite-cavity semiconductor lasers", IEEE Journal of Quantum Electronics, vol. 26, NO. 1, pp.32-44, 1990.

[16] A. P. Bogatov, P. G. Eliseev and B. N. Sverdlov "Anomalous interaction of spectral modes in a semiconductor laser", IEEE Journal of Quantum Electronics, vol. 11, NO. 7, pp.510-515, 1975. 
[17] G. P. Agrawal, "Population pulsations and nondegenerate four-wave mixing in semiconductor lasers and amplifiers", Journal of Optical Society of America B, vol. 5, NO.1, pp.302-304, 1988.

[18] G. P. Agrawal, "Highly nondegenerate four-wave mixing in semiconductor lasers due to spectral hole burning", Applied Physics Letters, vol. 5, pp.302-304, 1987.

[19] G. R. Gray and G. P. Agrawal, "Importance of self-induced carrier-density modulation in semiconductor lasers", IEEE Photonics Technology Letters, vol.4, pp. 1216-1219, 1992.

[20] M. Sargent III, M. P. Scully and W. E. Lamb, "Laser Physics", (Addison Wesley, Reading, Mass., USA), 1974.

[21] K. A. Shore, and W. M. Yee, "Theory of self-locking FM operation in semiconductor lasers", IEE proceedings-J, vol.138, No. 2, pp.91-96, 1991.

[22] I. Petitbon, P. Gallion, G. Debarge, and C. Chabran, "Locking bandwidth and relaxation oscillation of an injection-locked semiconductor laser", IEEE Journal of Quantum Electronics, vol.24, pp.148-154, 1988.

[23] C. H. Henry, "Theory of the phase noise and power spectrum of a single mode injection laser", IEEE Journal of Quantum Electronics, vol. 19, No. 9, pp.1391-1397, 1983.

[24] P. Spano, S. Piazzolla, and M. Tamburrini, "Phase noise in semiconductor lasers: a theoretical approach", IEEE Journal of Quantum Electronics, vol. 19, No. 7, pp.1195-1199, 1983. 
Table 1: Parameters used for calculations.

\begin{tabular}{|l|l|l|}
\hline Parameters \& Unit & Symbols & Values \\
\hline Width $[m]$ & $w$ & $1.5 \times 10^{-6}$ \\
\hline Thickness $[m]$ & $d$ & $0.3 \times 10^{-6}$ \\
\hline Total length $[m]$ & $L$ & $1.15 \times 10^{-3}$ \\
\hline Linewidth enhancement factor & $\alpha_{H}$ & 4 \\
\hline Optical confinement factor & $\Gamma$ & 0.06 \\
\hline Overlap factor & $C$ & 0.8 \\
\hline Gain group index & $n_{g}$ & 4 \\
\hline Phase and Bragg group indices & $n_{\varphi}, n_{B}$ & 3.3 \\
\hline Carrier lifetime $[s]$ & $\tau_{e}$ & $1.7 \times 10^{-9}$ \\
\hline Carrier density at transparency $\left[m^{-3}\right]$ & $N_{t r}$ & $1 \times 10^{24}$ \\
\hline Differential gain $\left[m^{2}\right]$ & $a$ & $9 \times 10^{-20}$ \\
\hline Total material losses $\left[\mathrm{cm}^{-1}\right]$ & $\gamma_{i n t} / v_{g}$ & 15 \\
\hline Spontaneous emission rate $\left[s^{-1}\right]$ & $R_{s p}$ & $5 \times 10^{12}$ \\
\hline Self-pulsation frequency $[G H z]$ & $\Omega_{s p} / 2 \pi$ & 42 \\
\hline
\end{tabular}


List of Figures 


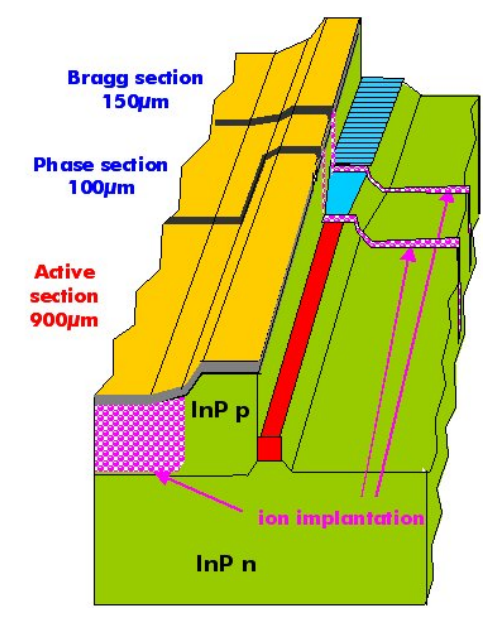

Figure 1: Schematic of the SP DBR laser. 
Figure 2: Optical spectrum measured for $I_{a}=\mathbf{2 3 0} \mathbf{~} \mathbf{A}$ and $I_{B}=120 \mathbf{~ m A}$. 
Figure 3: Electrical spectrum of photocurrent observed in the same experimental condition than that of Fig. 2 


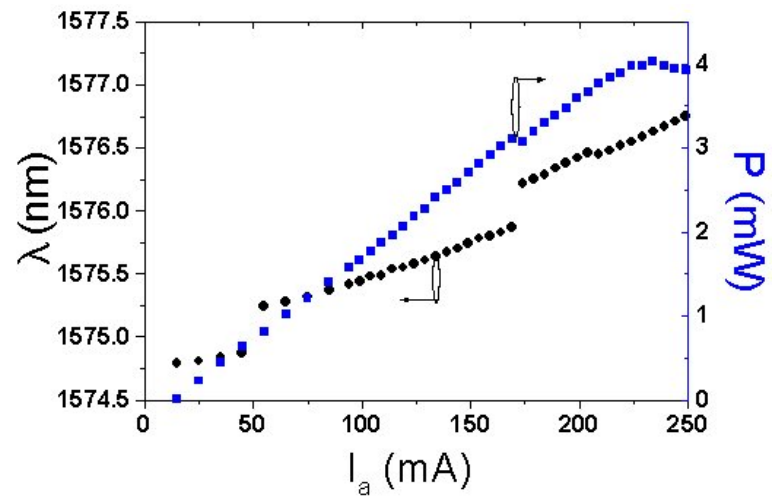

Figure 4: Wavelength and power evolutions of the main optical mode as a function of the active region current, with unbiased Bragg section. 


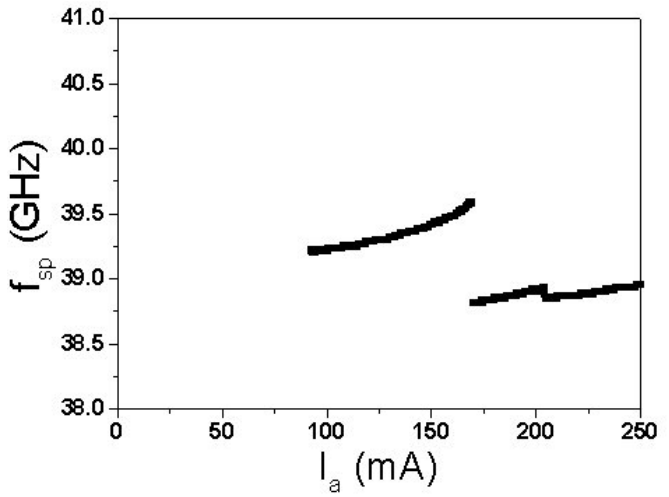

Figure 5: Evolution of the SP frequency versus the active current, with unbiased Bragg section. 
(a)

(b)

Figure 6: Wavelength evolution of the main optical mode as a function of the Bragg current, with an active current of $200 \mathrm{~mA}$ : (a) from 0 to $50 \mathrm{~mA}$ and (b) from 50 to $150 \mathrm{~mA}$. 
(a)

(b)

Figure 7: Evolution of the SP frequency versus the Bragg current, with an active current of 200 $\mathrm{mA}$ : (a) from 0 to $50 \mathrm{~mA}$ and (b) from 50 to $150 \mathrm{~mA}$. 
Figure 8: Evolution of the spectral linewidths of longitudinal modes and self-pulsation as a function of the Bragg current between two mode jumps. 
Figure 9: Radio frequency spectrum of the DBR laser with $I_{a}=$ and $I_{B}=$. 
(a)

(b)

Figure 10: Radio frequency spectrum of: (a) the beating component between modes $1 \& 2$ and (b) the beating component between modes $2 \& 3$. The inserts are the corresponding optical spectra. The bias conditions are identical than that of Fig.9 


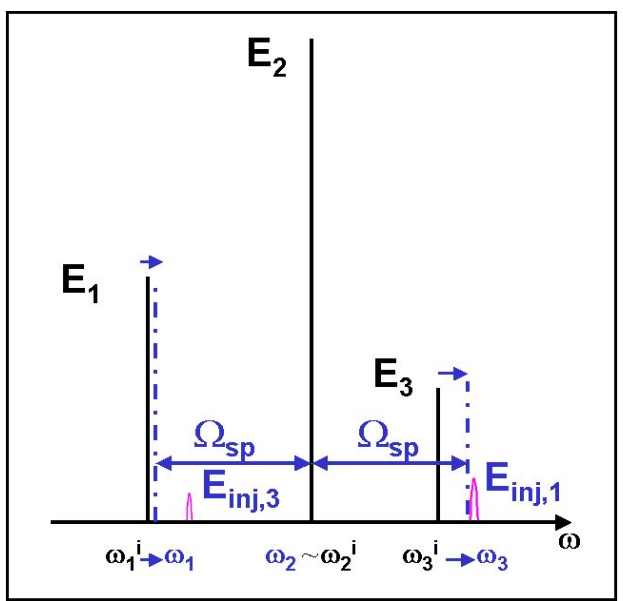

Figure 11: Scheme of mutual injection regime through modulation side-bands from FWM, leading to passively modes locked at $\Omega_{s p}$. 


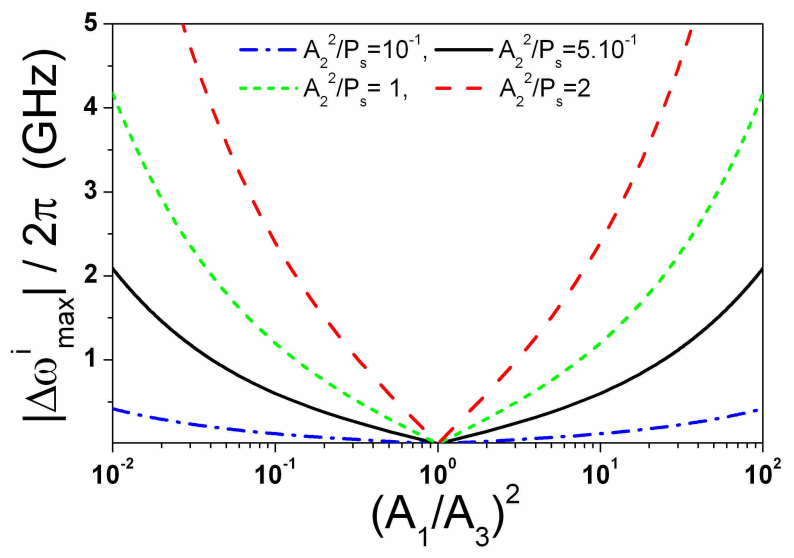

Figure 12: Evolution of the maximum initial frequency mismatch $\Delta \omega^{i} / 2 \pi$ versus the injection power rate, for different values of the ratio $A_{2}^{2} / P_{s}$. 


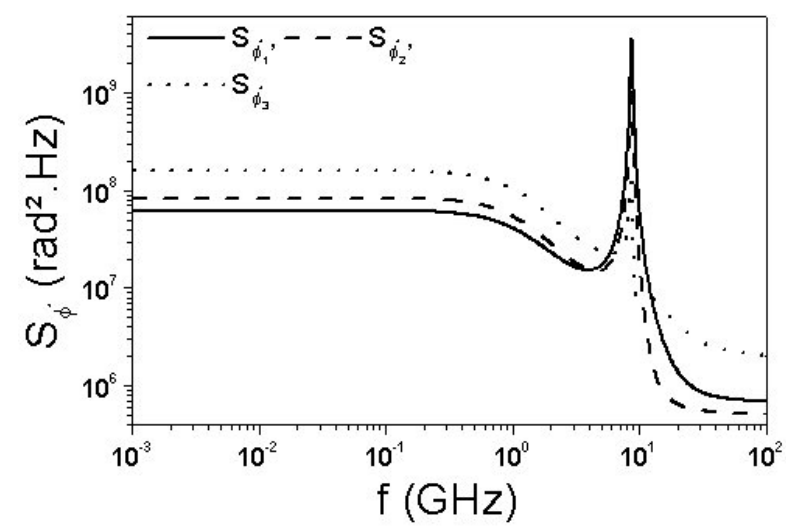

Figure 13: Numerical calculations of FM noise spectra for longitudinal modes. 


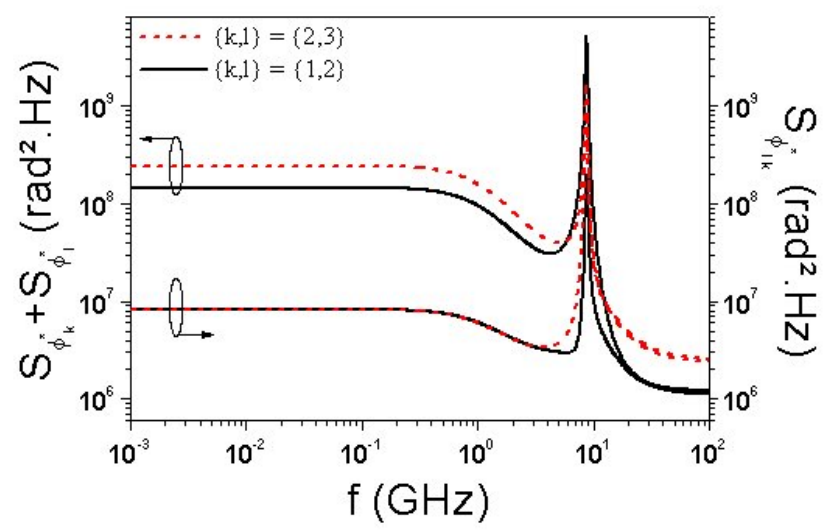

Figure 14: Numerical calculations of FM noise PSD for the relative phases $\phi_{21}$ and $\phi_{32}$, compared to the sum of those of longitudinal modes. 